neuropsychiatrie

vereinigt mit

psychiatrie \& psychotherapie

\title{
Erratum zu: Suizid und Suizidprävention 1938-1945 in Wien
}

\author{
Gernot Sonneck, Hans Hirnsperger, Reinhard Mundschütz \\ Online publiziert: 22. Februar 2013 \\ (c) Springer-Verlag Wien 2013
}

\section{Erratum zu: Neuropsychiatr (2012) 26:111-120 DOI 10.1007/s4021 1-012-0032-8}

Im oben stehenden Beitrag sind leider folgende Fehler aufgetreten:

In Abschnitt „Die Situation der jüdischen Bevölkerung“, 4. Absatz, Zeile 3 muss es lauten:... starben in Wien 50 Juden durch Suizid [14a].

In der Literatur, nach Stelle 14, ist folgendes Zitat einzufügen: 14a. Dokumentationsarchiv des österreichischen Widerstandes (Hrsg.). Die österreichischen Opfer des Holocaust. Wien: DÖW - Dokumentationsarchiv des österreichischen Widerstandes; 2001.

Korrespondenzadresse

\section{H. Hirnsperger}

Medizinische Universität Wien

Zentrum für Public Health

Institut für medizinische Psychologie

Kinderspitalgasse 15/Parterre

1090 Wien

hans.hirnsperger@meduniwien.ac.at

Die Onlineversion des Originalbeitrages ist erreichbar unter doi:http://dx.doi.org/10.1007/s40211-012-0032-8

\author{
H. Hirnsperger $(\bowtie)$ \\ Zentrum für Public Health, Institut für medizinische Psychologie, \\ Medizinische Universität Wien, Kinderspitalgasse 15/Parterre, \\ 1090 Wien, Österreich \\ E-Mail: hans.hirnsperger@meduniwien.ac.at \\ G. Sonneck \\ Institut für medizinische Psychologie, Zentrum für Public Health, \\ Medizinische Universität Wien, Severingasse 9, \\ 1090 Wien, Österreich \\ G. Sonneck \\ Ludwig Boltzmann-Institut für Sozialpsychiatrie, \\ Lazarettgasse 14A-912, 1090 Wien, Österreich \\ R. Mundschütz \\ Universitätsbibliothek der Medizinischen Universität Wien, \\ Währinger Gürtel 18-20, 1097 Wien, Österreich
}

